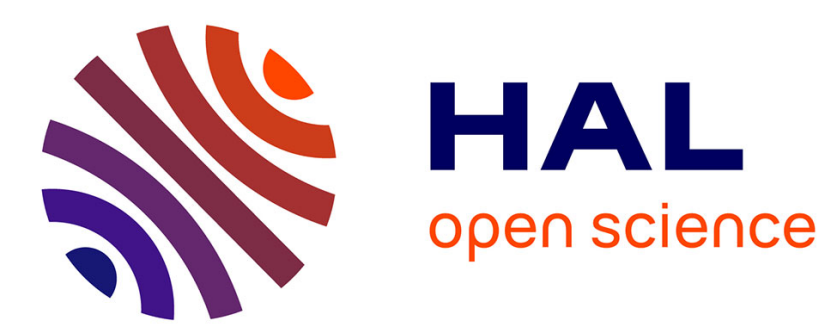

\title{
Episodes of particle ejection from the surface of the active asteroid (101955) Bennu
}

\author{
D. Lauretta, C. Hergenrother, S. Chesley, J. Léonard, J. Pelgrift, C. Adam, \\ M. Al Asad, P. Antreasian, R.-L. Ballouz, K. Becker, et al.
}

\section{To cite this version:}

D. Lauretta, C. Hergenrother, S. Chesley, J. Léonard, J. Pelgrift, et al.. Episodes of particle ejection from the surface of the active asteroid (101955) Bennu. Science, 2019, 366 (6470), pp.eaay3544. 10.1126/science.aay3544 . hal-02407783

\section{HAL Id: hal-02407783 https://hal.science/hal-02407783}

Submitted on 21 Dec 2020

HAL is a multi-disciplinary open access archive for the deposit and dissemination of scientific research documents, whether they are published or not. The documents may come from teaching and research institutions in France or abroad, or from public or private research centers.
L'archive ouverte pluridisciplinaire HAL, est destinée au dépôt et à la diffusion de documents scientifiques de niveau recherche, publiés ou non, émanant des établissements d'enseignement et de recherche français ou étrangers, des laboratoires publics ou privés. 


\section{RESEARCH ARTICLE SUMMARY}

\section{ASTEROIDS}

\section{Episodes of particle ejection from the surface of the active asteroid (101955) Bennu}

\author{
D. S. Lauretta* ${ }^{*}$ and C. W. Hergenrother ${ }^{*} \dagger$ et al.
}

INTRODUCTION: Active asteroids are small bodies in the Solar System that show ongoing mass loss, such as the ejection of dust, which may be caused by large impacts, volatile release, or rotational acceleration. Studying them informs our understanding of the evolution and destruction of asteroids and the origin of volatile materials such as water on Earth.

The OSIRIS-REx (Origins, Spectral Interpretation, Resource Identification, and SecurityRegolith Explorer) spacecraft has rendezvoused with the near-Earth asteroid (101955) Bennu. The selection of Bennu as the OSIRIS-REx target was partially based on its spectral similarity to some active asteroids. Observations designed to detect mass loss at Bennu were conducted from Earth and during the space- craft's approach, but no signs of asteroid activity were found. However, when the spacecraft entered orbit in January 2019, we serendipitously observed particles in the vicinity of Bennu that had apparently been ejected from its surface.

RATIONALE: We analyzed the properties and behavior of particles ejected from Bennu to determine the possible mechanisms of ejection and provide understanding of the broader population of active asteroids. Images obtained by the spacecraft indicate multiple discrete ejection events with a range of energies and resultant particle trajectories. We characterized three large ejection events that respectively occurred on 6 January, 19 January, and 11 February 2019. Tracking of individual

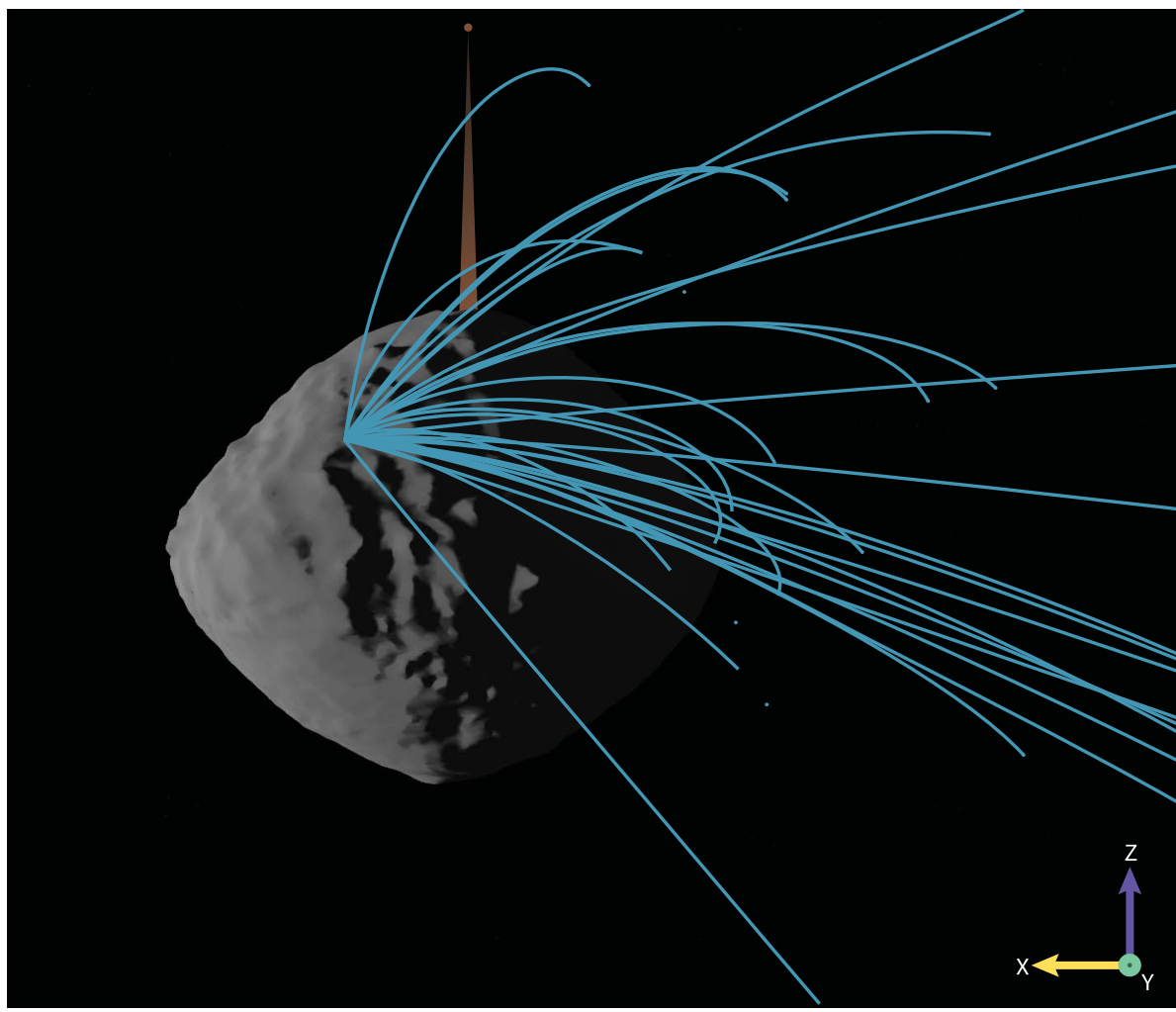

Schematic diagram of orbit determination model output for the 19 January 2019 particle ejection event from asteroid Bennu observed by the OSIRIS-REx spacecraft. Bennu is depicted in gray and has a diameter of $\sim 500 \mathrm{~m}$. OSIRIS-REx is indicated with the brown dot, $2 \mathrm{~km}$ from Bennu's center of mass; the cone represents the viewing angle. Blue arcs are particle trajectories, ending or with gaps where the trajectories pass into shadow. The Sun-angular momentum frame coordinates are shown at bottom right: $x$, solar vector; $y$, Bennu orbital direction; $z$, Bennu north.

particles across multiple images by means of optical navigation techniques provided the initial conditions for orbit determination modeling. By combining these approaches, we estimated the locations and times of ejection events and determined initial velocity vectors of particles. We estimated the particle sizes and the minimum energies of the ejection events using a particle albedo and density consistent with observations of Bennu.

RESULTS: Particles with diameters from $<1$ to $\sim 10 \mathrm{~cm}$ were ejected from Bennu at speeds ranging from $\sim 0.05$ to $>3 \mathrm{~m} \mathrm{~s}^{-1}$. Estimated energies ranged from $270 \mathrm{~mJ}$ for the 6 January event to $8 \mathrm{~mJ}$ for the 11 February event. The

\section{ON OUR WEBSITE}

Read the full article at http://dx.doi org/10.1126/ science.aay3544

these events all occurred 18:00 local solar time.

In addition to discrete ejection events, we detected a persistent background of particles in the Bennu environment. Some of these background particles have been observed to persist on temporary orbits that last several days-in one case, with a semimajor axis $>1 \mathrm{~km}$. The orbital characteristics of these gravitationally bound objects make it possible to determine the ratio of their cross-sectional area to their mass. Combined with their photometric phase functions, this information constrains the parameter space of the particles' diameters, densities, and albedos.

CONCLUSION: Plausible mechanisms for the large ejection events include thermal fracturing, volatile release through dehydration of phyllosilicates, and meteoroid impacts. The late-afternoon timing of the events is consistent with any of these mechanisms. Bennu's boulder geology indicates that thermal fracturing, perhaps enhanced by volatile release, could occur on the asteroid surface. Smaller events, especially those that occur on the night side of Bennu, could be attributable to reimpacting particles.

Our observations classify Bennu as an active asteroid. Active asteroids are commonly identified by major mass loss events observable with telescopes, on scales much greater than we observed at Bennu. Our findings indicate that there is a continuum of mass loss event magnitudes among active asteroids.

The full lists of author names and affiliations are available in the full article online.

*Corresponding author. Email: lauretta@orex.Ipl.arizona.edu (D.S.L.); chergen@Ipl.arizona.edu (C.W.H.)

tThese authors contributed equally to this work.

Cite this article as D. S. Lauretta and C. W. Hergenrother et al., Science 366, eaay3544 (2019). DOI: 10.1126/science.aay3544. 


\section{RESEARCH ARTICLE}

ASTEROIDS

\section{Episodes of particle ejection from the surface of the active asteroid (101955) Bennu}

D. S. Lauretta ${ }^{1 *}$, , C. W. Hergenrother ${ }^{1 *}$, , S. R. Chesley ${ }^{2}$, J. M. Leonard ${ }^{3}$, J. Y. Pelgrift ${ }^{3}$, C. D. Adam ${ }^{3}$, M. Al Asad ${ }^{4}$, P. G. Antreasian ${ }^{3}$, R.-L. Ballouz ${ }^{1}$, K. J. Becker ${ }^{1}$, C. A. Bennett ${ }^{1}$, B. J. Bos ${ }^{5}$, W. F. Bottke ${ }^{6}$, M. Brozović ${ }^{2}$, H. Campins ${ }^{7}$, H. C. Connolly Jr. 8,1 , M. G. Daly ${ }^{9}$, A. B. Davis ${ }^{10}$, J. de León ${ }^{11}$, D. N. DellaGiustina ${ }^{1,12}$, C. Y. Drouet d'Aubigny'1, J. P. Dworkin ${ }^{5}$, J. P. Emery ${ }^{13,14}$, D. Farnocchia², D. P. Glavin ${ }^{5}$, D. R. Golish ${ }^{1}$, C. M. Hartzell ${ }^{15}$, R. A. Jacobson ${ }^{2}$, E. R. Jawin ${ }^{16}$, P. Jenniskens ${ }^{17}$, J. N. Kidd Jr. ${ }^{1}$, E. J. Lessac-Chenen ${ }^{3}$, J.-Y. Li ${ }^{18}$, G. Libourel ${ }^{19}$, J. Licandro ${ }^{11}$, A. J. Liounis ${ }^{5}$, C. K. Maleszewski ${ }^{1}$, C. Manzoni ${ }^{20}$, B. May ${ }^{20}$, L. K. McCarthy ${ }^{3}$, J. W. McMahon ${ }^{10}$, P. Michel ${ }^{19}$, J. L. Molaro ${ }^{18}$, M. C. Moreau ${ }^{5}$, D. S. Nelson ${ }^{3}$, W. M. Owen Jr. ${ }^{2}$, B. Rizk' ${ }^{1}$ H. L. Roper ${ }^{1}$, B. Rozitis ${ }^{21}$, E. M. Sahr ${ }^{3}$, D. J. Scheeres ${ }^{10}$, J. A. Seabrook ${ }^{9}$, S. H. Selznick ${ }^{1}$, Y. Takahashi' ${ }^{2}$, F. Thuillet ${ }^{19}$, P. Tricarico ${ }^{18}$, D. Vokrouhlickýn ${ }^{22}$, C. W. V. Wolner ${ }^{1}$

Active asteroids are those that show evidence of ongoing mass loss. We report repeated instances of particle ejection from the surface of (101955) Bennu, demonstrating that it is an active asteroid. The ejection events were imaged by the OSIRIS-REx (Origins, Spectral Interpretation, Resource Identification, and Security-Regolith Explorer) spacecraft. For the three largest observed events, we estimated the ejected particle velocities and sizes, event times, source regions, and energies. We also determined the trajectories and photometric properties of several gravitationally bound particles that orbited temporarily in the Bennu environment. We consider multiple hypotheses for the mechanisms that lead to particle ejection for the largest events, including rotational disruption, electrostatic lofting, ice sublimation, phyllosilicate dehydration, meteoroid impacts, thermal stress fracturing, and secondary impacts.

A ctive asteroids are small bodies that have typical asteroidal orbits but show some level of mass-loss activity, such as ejection of dust or the development of a coma or tail (1). Several objects in the main asteroid belt or the near-Earth asteroid population have been observed to show vary-

'Lunar and Planetary Laboratory, University of Arizona, Tucson, AZ, USA. ${ }^{2}$ Jet Propulsion Laboratory, California Institute of Technology, Pasadena, CA, USA. ${ }^{3}$ KinetX Aerospace, Simi Valley, CA, USA. ${ }^{4}$ Department of Earth, Ocean, and Atmospheric Sciences, University of British Columbia, Vancouver, BC, Canada. ${ }^{5}$ NASA Goddard Space Flight Center, Greenbelt, MD, USA. ${ }^{6}$ Southwest Research Institute, Boulder, CO, USA. ${ }^{7}$ Department of Physics, University of Central Florida, Orlando, FL, USA. ${ }^{8}$ Department of Geology, Rowan University, Glassboro, NJ, USA ${ }^{9}$ The Centre for Research in Earth and Space Science, York University, Toronto, ON, Canada. ${ }^{10}$ Smead Department of Aerospace Engineering Sciences, University of Colorado, Boulder, CO, USA. ${ }^{11}$ Instituto de Astrofísica de Canarias and Departamento de Astrofísica, Universidad de La Laguna, Tenerife, Spain.

${ }^{12}$ Department of Geosciences, University of Arizona, Tucson, AZ, USA. ${ }^{13}$ Department of Earth and Planetary Sciences, University of Tennessee, Knoxville, TN, USA. ${ }^{14}$ Department of Astronomy and Planetary Sciences, Northern Arizona University, Flagstaff, AZ, USA. ${ }^{15}$ Department of Aerospace Engineering, University of Maryland, College Park, MD, USA. ${ }^{16}$ Smithsonian Institution National Museum of Natural History, Washington, DC, USA. ${ }^{17}$ SET (Search for Extraterrestrial Intelligence) Institute, Mountain View, CA USA ${ }^{18}$ Planetary Science Institute, Tucson, AZ, USA.

${ }^{19}$ Université Côte d'Azur, Observatoire de la Côte d'Azur, CNRS (Centre national de la recherche scientifique), Laboratoire Lagrange, Nice, France. ${ }^{20}$ London Stereoscopic Company, London, UK. ${ }^{21}$ School of Physical Sciences, Open University, Milton Keynes, UK. ${ }^{22}$ Institute of Astronomy, Charles University, Prague, Czech Republic

*Corresponding author. Email: lauretta@orex.Ipl.arizona.edu (D.S.L.); chergen@Ipl.arizona.edu (C.W.H.) †These authors contributed equally to this work. ing levels of mass loss, such as the active asteroid 133P/Elst-Pizarro (2). Some of these objects behave as comets and eject dust over long periods of time, from days to months, or during multiple perihelion passages [including 133P/Elst-Pizarro (3)]. Other active asteroids eject dust over short time scales in one or a series of impulsive events, such as in the case of (6478) Gault (4). Still others have been observed to split into multiple objects or, in the case of P/2016 G1 (PANSTARRS), completely disintegrate (5). Near-Earth asteroid (3200) Phaethon has exhibited low levels of mass loss during multiple orbits when less than 0.15 astronomical units (AU) from the Sun $(6,7)$. Multiple ejection mechanisms have been suggested to explain asteroid activity, including collisions, water-ice sublimation, rotational destabilization, thermal fracturing, and dehydration (8).

The OSIRIS-REx (Origins, Spectral Interpretation, Resource Identification, and Security500-m-diameter B-type near-Earth asteroid (101955) Bennu in December 2018. Bennu was selected as the mission target partly because of its spectral similarity to some active asteroids (9). Here, we describe and analyze OSIRIS-REx observations of activity originating from Bennu's surface. We initially detected this phenomenon in navigational images from 6 January 2019, 1 week after the spacecraft entered orbit and 4 days before Bennu periRegolith Explorer) spacecraft arrived at the helion (10). We subsequently detected multiple particle ejection events between December 2018 and February 2019. The largest observed events each released dozens of observed particles.

\section{Particle detections}

Dust and natural satellite searches were conducted during the spacecraft's approach to Bennu during early proximity operations in September to November 2018, which yielded null results (10). Signs of asteroid activity may have been detected by the OSIRIS-REx Laser Altimeter [OLA (11)] (figs. S1 and S2) in December 2018. OLA recorded 21 lidar returns off the limb of the asteroid during the Preliminary Survey mission phase, including four at distances of $399 \mathrm{~m}$ (4 December), $397 \mathrm{~m}$ (8 December), and 562 and $576 \mathrm{~m}$ (12 December, 3.1 hours apart) from Bennu's center. These four signals prompted a search for corresponding objects in images from the same dates, without success. However, the geometry suggests that these four returns were probably from objects or groups of objects (12). The earliest evidence of activity in imaging data is a particle $8 \pm 3 \mathrm{~cm}(1 \sigma)$ in diameter on a suborbital trajectory, imaged by the NavCam 1 imager of the Touch and Go Camera System (TAGCAMS) (13) on 10 December 2018. We cannot rule out activity before December 2018. The searches performed during the spacecraft's approach to the asteroid did not have sufficient sensitivity to detect most of the activity that was later observed at closer ranges. A particle as large as the one observed on 10 December would have been detectable with the natural satellite searches; lack of detection implies that events ejecting particles of that size were relatively rare or nonexistent during the spacecraft's approach.

On 31 December 2018, the spacecraft entered into an eccentric, near-terminator orbit that ranged between 1.6 and $2.1 \mathrm{~km}$ from Bennu's center of mass. This Orbital A mission phase continued until 28 February 2019, when the spacecraft departed orbit to perform the Detailed Survey (14). During the early part of Orbital A, we acquired NavCam 1 image sets roughly every 2 hours to provide optical navigation (OpNav) data for the flight dynamics team (table S1) (15). Each image set consisted of four images taken in pairs $\sim 7$ min apart. Each pair contained a short-exposure image (1.4 ms) to capture landmarks on Bennu's surface, followed immediately by a long-exposure image ( $5 \mathrm{~s}$ ) to capture the background star field.

The first particle ejection event that we identified was observed in OpNav images taken on 6 January at 20:56:21 Coordinated Universal Time (UTC) (Fig. 1, A and B, and fig. S3). The particles appear as more than 200 starlike point-source objects and trailed (highervelocity) objects located off the northern polar limb of Bennu. The image taken $7 \mathrm{~min}$ and $16 \mathrm{~s}$ 

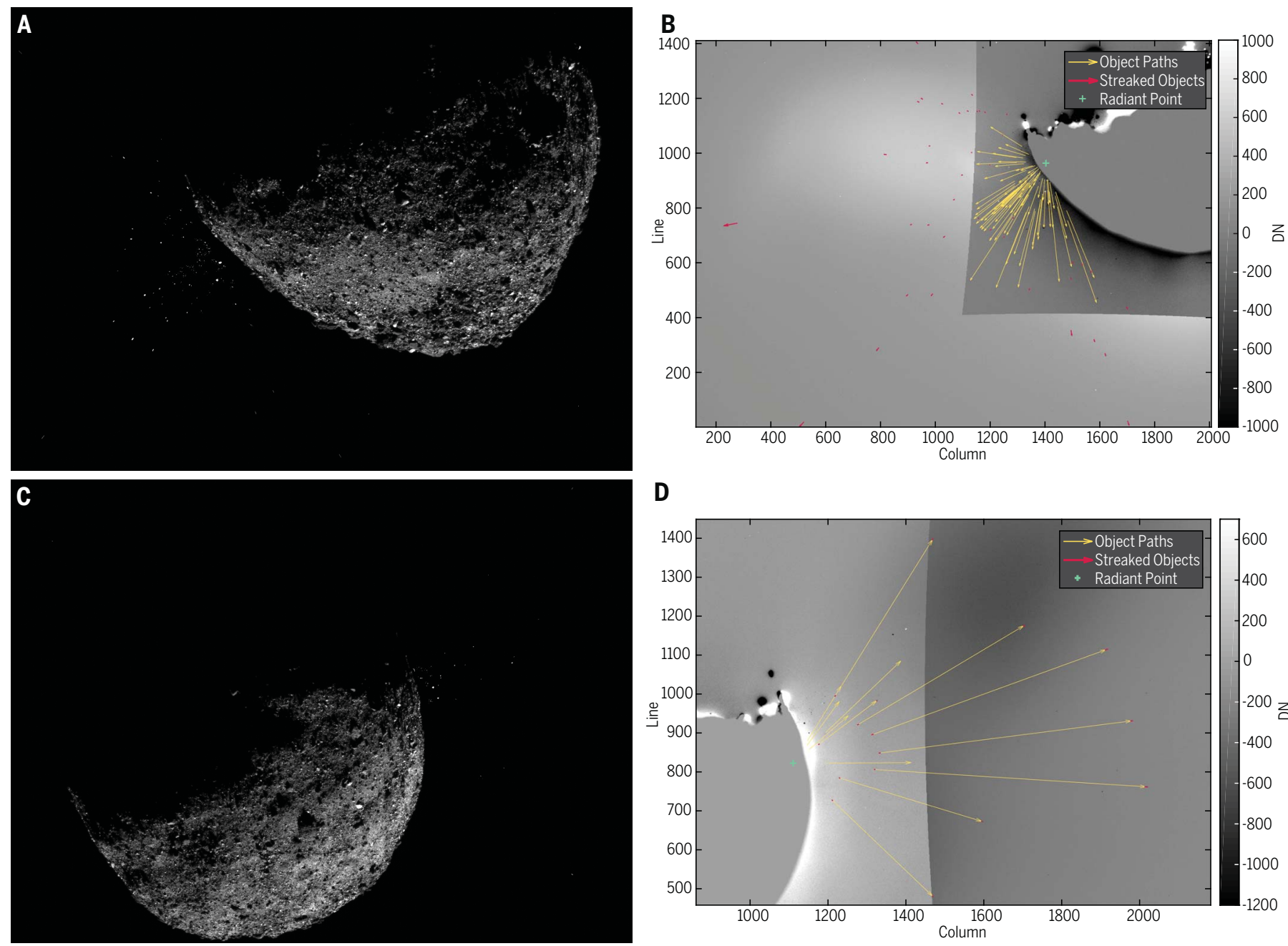

\section{D}

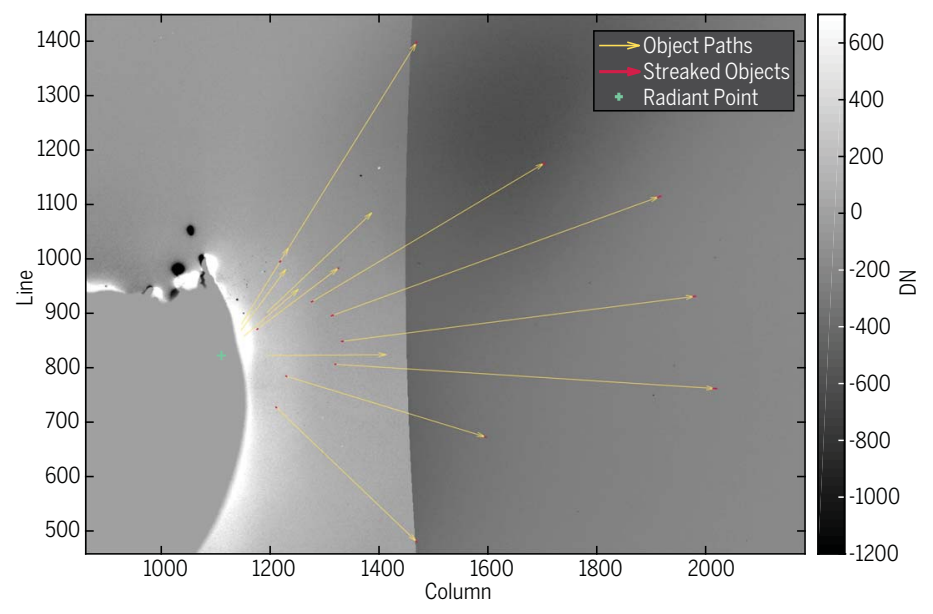

Fig. 1. Particle ejections from Bennu. (A and $\mathbf{C})$ Composite views of particle ejections from the surface of asteroid Bennu on (A) 6 January and (C)

19 January. These images were produced by combining two exposures taken by the NavCam 1 imager in immediate succession: a short-exposure image $(1.4 \mathrm{~ms})$, showing the asteroid, and a long-exposure image ( $5 \mathrm{~s}$ ), showing the particles (12). Image processing techniques were applied to increase the brightness and contrast of the ejected particles, which would otherwise be invisible at the same time as the bright asteroid surface (12). The original images are shown in fig. S3. In (A), Bennu's north $(+z)$ pole is to the top right, pointing into the image; the subobserver latitude is $-36^{\circ}$. In $(C)$, the north pole is to the top right, pointing out of the image; the subobserver latitude is $60^{\circ}$. (B and $\mathbf{D}$ ) Two NavCam images taken immediately after the ejection events on (B) 6 January and (D) 19 January are registered on the center of Bennu and differenced to

highlight any moving particles. Particles moving at high velocity appear as streaks in a single image (red) that provide position information at the start and end of the exposure. The paths of particles moving more slowly (yellow) are identified from individual particles detected in the earlier image that also are present in the later image, farther from Bennu's limb. For each event, the apparent motion of the individual particles traces back to a radiant point on Bennu's surface (light blue cross) that indicates the potential source region on the near side of Bennu. A second possible source region occurs on the far side of Bennu, out of view. The shaded area closest to the asteroid [darker shading in $(B)$, lighter shading in (D)] corresponds to where the two images share a common field of view and are differenced. The opposite shading corresponds only to the image with the larger field of view [earlier image in (B), later image in (D)]. DN, data number.

later shows objects in common with the earlier image that have moved away from Bennu, implying the movement of discrete particles (Fig. 1B). This observation triggered an immediate risk assessment of whether it was safe for the spacecraft to remain in orbit, which was concluded affirmatively, and led to an observational campaign to detect and characterize Bennu's apparent activity.

We increased the imaging cadence in response to the initial event to better characterize the frequency of particle ejections and any persistent particle population (table S1). Starting on 11 January, NavCam 1 began collecting image pairs of each field every $30 \mathrm{~min}$. On 28 January, we again increased the cadence, collecting image pairs of each field every $20 \mathrm{~min}$. This imaging frequency continued until 18 February. During this time period, we detected two additional ejection events of a similar scale, on 19 January (Fig. 1, C and D, and fig. S3) and 11 February (fig. S4). The distance from the spacecraft to Bennu's center of mass was $1.66 \mathrm{~km}$ for 6 January, $1.99 \mathrm{~km}$ for 19 January, and $1.64 \mathrm{~km}$ for 11 February. We used the imaging dataset to characterize these three events, which were the largest observed (they had the highest number of detected particles). We also observed several smaller events, in which fewer than 20 particles were detected (Fig. 2). There is also a persistent background level of particles in the Bennu environment; we detected a few particles per day during Orbital A, with observed increases immediately after the 19 January and 11 February events (Fig. 2). 


\section{Characterization of the largest observed events}

For the 6 January, 19 January, and 11 February events, a particle distribution pattern near the limb of Bennu in the first image of each event is also apparent in the image collected $\sim 7 \mathrm{~min}$ later, farther from the limb and dispersed (Fig. $1, \mathrm{~B}$ and D), and also appears in subsequent images for the 19 January and 11 February events. Using OpNav techniques developed for spacecraft navigation, we associated individual particle detections from this pattern and determined the trajectory and velocity of each particle (12). Fast-moving particles cross multiple pixels in a single exposure and appear as trails, providing position and velocity information within one image. For each event, OpNav analysis constrains two possible locations (a near and far radiant) on Bennu's surface from which the particles originated (Fig. 3 , Table 1, and table S2) (12).

The 6 January event is the least constrained (particles detected in only two images) of the three largest events. We determined that the event originated at a high southern latitude (between about $57^{\circ} \mathrm{S}$ and $75^{\circ} \mathrm{S}$ ) (Table 1 and Fig. 3A) (12), with an ejection time between 15:22 and 16:35 local solar time (LST). However, the event location relative to the spacecraft and the limited dataset make estimating the precise latitude and ejection time difficult. For this event, we determined speeds for 117 of the 200 observed particles, ranging from 0.07 to $3.3 \mathrm{~m} \mathrm{~s}^{-1}$. Fifty-two particles were moving more slowly than Bennu's escape velocity $\left[20 \mathrm{~cm} \mathrm{~s}^{-1}\right.$ for the volume-averaged Bennu radius $(12,16)]$ (fig. $\mathrm{S} 5)$.

Because of the increased imaging cadence, there is a more extensive dataset for the 19 January and 11 February events. We used the output of the OpNav characterization to provide initial conditions for higher-fidelity orbit determination (OD) modeling. In these models, we assumed that the particles from a given event left Bennu's surface at the same location on a trajectory influenced by pointmass gravity (12). We performed this analysis on 24 particles from the 19 January event (Movie 1) and 25 particles from the 11 February event. For these two events, with individual particles identified in more than three images, this analysis allows us to estimate a single location for the particle source location (Fig. 3, $\mathrm{B}$ and $\mathrm{C}$ ) as well as ejection timing and initial velocity vectors (Table 1 ).

We determined the ejection epoch (moment in time) by extrapolating the OD solutions backward to the point where they intersect Bennu's surface. This analysis shows that the event on 19 January occurred at 00:53:41 \pm $4 \mathrm{~s}(3 \sigma)$ UTC from a location on Bennu at latitude $20^{\circ} \mathrm{N}$, longitude $335^{\circ}$. The epoch corresponds to 16:38 LST at that location. Surface ejection velocity magnitudes ranged from 0.06 to $1.3 \mathrm{~m} \mathrm{~s}^{-1}$. The 19 January timing data show a bimodal distribution, with a small peak occurring 6 min before the main epoch (fig. S6), suggesting that some of

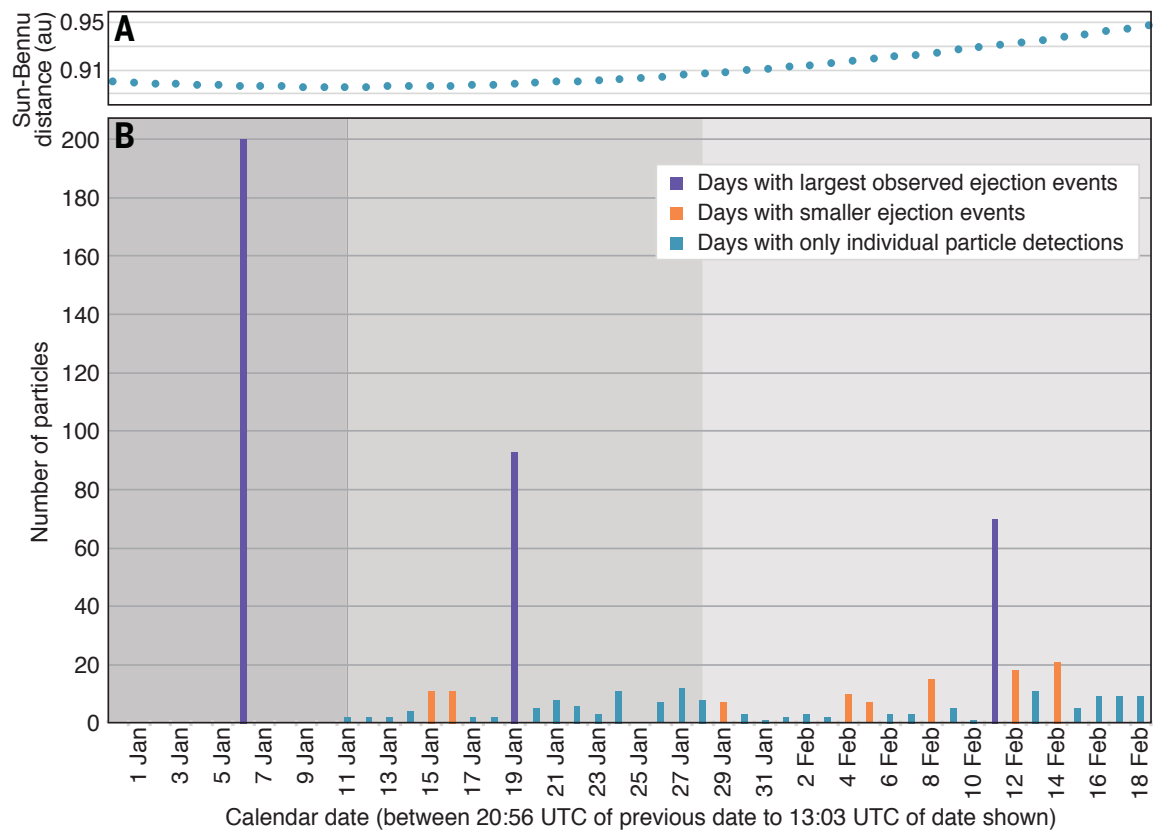

Fig. 2. Particle detections during the Orbital A mission phase. (A) Distance of Bennu from the Sun over the same time period as shown in (B). (B) Particle detections associated (purple and orange) and not associated (light blue) with observed ejection events. The changes in the background shading indicate when the observation cadence increased on 11 January and again on 28 January 2019. the particles may have ejected in a smaller event before the large release. The event on 11 February occurred at 23:27:28 $\pm 6 \mathrm{~s}(3 \sigma)$ UTC from latitude $20^{\circ} \mathrm{N}$, longitude $60^{\circ}$, corresponding to 18:05 LST, with observed velocity magnitudes ranging from 0.07 to $0.21 \mathrm{~m} \mathrm{~s}^{-1}$. All particles from this event appear to have left the surface nearly simultaneously (fig. S6). Many of the characterized particles are on ballistic trajectories that reimpact the surface on the night side of Bennu, whereas highvelocity particles escape on hyperbolic trajectories (Movie 1).

Images of the particle source locations on Bennu (Fig. 3, A to C) show no obvious geological distinction from other locations on the surface of Bennu. The event radiant locations contain abundant rocks that are diverse in size and surface texture, as well as small circular depressions that may be impact craters. However, similar features are globally distributed on Bennu $(17,18)$. We analyzed the normal albedo distribution of the two better constrained source regions (19 January and 11 February) and found that they are similar to the global distribution for Bennu (19), averaging $0.042 \pm 0.003(1 \sigma)$ (Fig. 3, D and E) (12). The lack of obvious morphologic or albedo variation may be due to the very low energies associated with the ejection events (Table 1 and table S3).

\section{Characterization of gravitationally bound particles}

In addition to particles released in ejection events, we observed a gravitationally bound background population of particles in the Bennu environment (Fig. 2). Among these are a few objects that remain in orbit for several days. From among the 215 tracks (linkages of individual detections of the same particle over a short time), we identified a representative group of six distinct gravitationally bound particles for further analysis. The trajectories around Bennu of these six particles and their altitude histories are shown in Fig. 4. Orbital elements are given in table S4 and fig. S7. Particles 1 to 4 are on short-lived orbits, persisting for 4 to 17 revolutions, with lifetimes ranging from 2 to 6 days. These orbits show a range of inclinations, from near equatorial to polar. Both prograde and retrograde orbits occur. The semimajor axis of particle 1 is $>1 \mathrm{~km}$, compared with 0.4 to $0.5 \mathrm{~km}$ for particles 2 to 4 . Particles 5 and 6 are suborbital. By extrapolating the orbits back to the time when they intersected Bennu's surface, we determined that three of the six particles ejected from the night side of Bennu (between 18:00 and 06:00 LST) (table S5). The six particles were ejected with orbital velocities in the range of 15 to $20 \mathrm{~cm} \mathrm{~s}^{-1}$. Surfacerelative velocities at ejection range from roughly 10 to $25 \mathrm{~cm} \mathrm{~s}^{-1}$. 

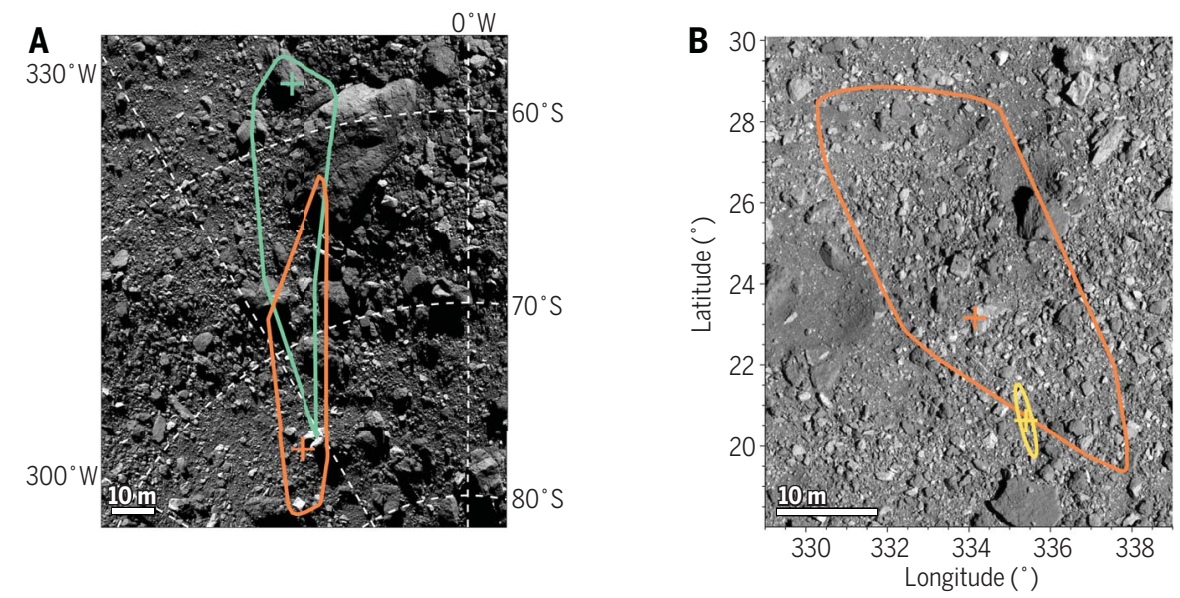

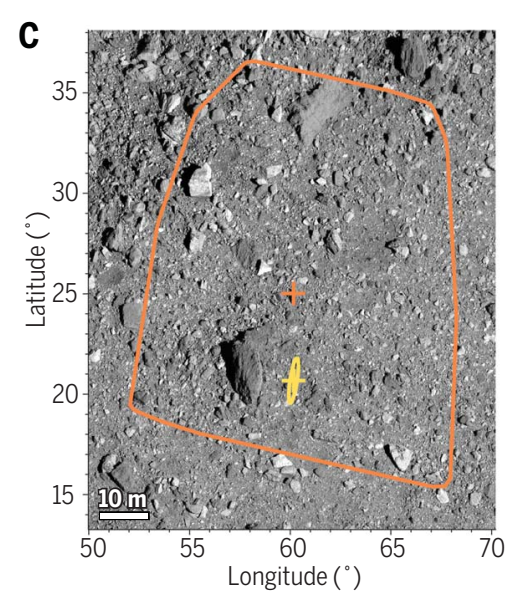

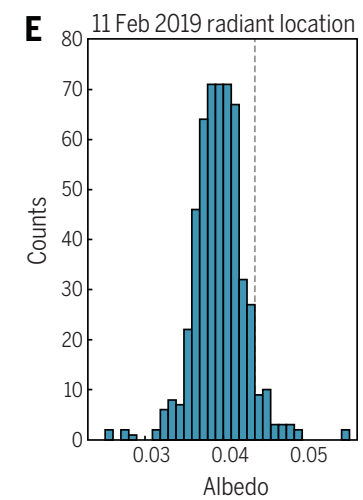

Fig. 3. Ejection source regions and their surface albedo distributions.

(A to C) Radiant locations of the (A) 6 January, (B) 19 January, and (C) 11 February particle ejection events are overlain on a mosaic of Bennu (12). Orange and teal crosses indicate the far and near candidate radiant locations, respectively, determined from OpNav analysis; orange and teal outlines enclose the $3 \sigma$ uncertainty region. The yellow crosses indicate the most likely source location determined from OD analysis; yellow lines trace the $3 \sigma$ uncertainty. (D and $\mathbf{E}$ ) For the locations of the latter two events, which are more tightly constrained, we show the surface albedo distributions (radiant locations with $3 \sigma$ uncertainties) (12). The dashed vertical lines indicate the average albedo of Bennu's surface (19).

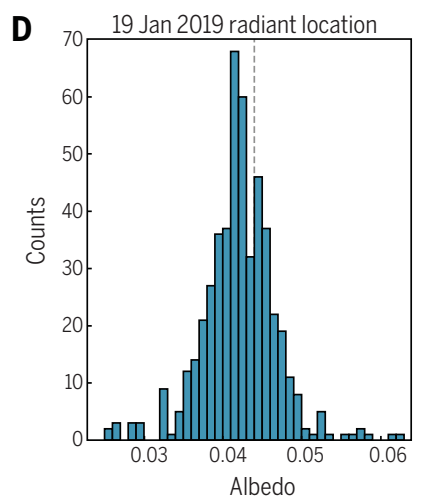

\section{Particle properties}

We constrained the area-to-mass ratios (where area is cross-sectional) of the six bound particles by using the trajectory information and modeling the nongravitational forces, which primarily arise from radiation pressures (table S5) (12). The particle trajectories also enabled us to calculate the phase angle and range of each observation to the spacecraft, from which we determined the photometric phase functions for particles 1 to 3 , constraining the visible absolute magnitude of each particle (table S5). Combining the area-to-mass ratio and absolute magnitude information, and assuming a spherical shape, defines a distinct curve in density $(\rho)$-albedo $\left(p_{\mathrm{V}}\right)$ space for each particle (fig. S8). If we further assume particles with densities of $2 \mathrm{~g} \mathrm{~cm}^{-3}$ [on the basis of Bennu meteorite analogs (20)], then their normal albedos range from 0.05 to 0.3 . In that case, the derived albedos are brighter than $96 \%$ of the material on Bennu, and the particle diameters range from 0.4 to $4.4 \mathrm{~cm}$. If, on the other hand, the particles have normal albedos of 0.04, which is consistent with the average surface material on Bennu (19), then the densities range from $0.7 \pm 0.3(1 \sigma)$ to $1.7 \pm 0.4(1 \sigma) \mathrm{g} \mathrm{cm}^{-3}$ (fig. S8). The high end of this range is consistent with meteorite analogs. The lower densities lead to larger particle diameters, ranging from 1.2 to $8.5 \mathrm{~cm}$. Given these uncertainties, we conclude that the particle diameters are in the range of $<1$ to $\sim 10 \mathrm{~cm}$.

With these constraints on the particle sizes, and the ejection velocities from the $\mathrm{OD}$ analysis, we can estimate the energy of the ejection events (Table 1 and table S3) (12). Such estimates should be considered lower limits because we may not have observed all ejected particles. In addition, our calculation assumes that the ejected particles had the average surface albedo of Bennu (0.044) (table S3) and the meteorite analog density of $2 \mathrm{~g} \mathrm{~cm}^{-3}$. For 6 January, the 124 particles with measured photometry ranged in size from $<1$ to $8 \mathrm{~cm}$, yielding a minimum event energy of $\sim 270 \mathrm{~mJ}$. For the 19 January event, more than 93 photometrically measured particles with radii between $<1$ and $7 \mathrm{~cm}$ ejected from the surface, giving a minimum event energy of $100 \mathrm{~mJ}$. For 11 February, more than 60 particles with radii between $<1$ and $7 \mathrm{~cm}$ ejected from the surface, with an associated minimum event energy of $8 \mathrm{~mJ}$ (uncertainties on the event energies are provided in Table 1).

\section{Possible ejection mechanisms}

Several constraints apply to the particle ejection mechanism: The three largest observed ejection events occurred in the late afternoon, between 15:22 and 18:05 LST. The largest observed event (6 January) occurred days before
Bennu reached perihelion (Fig. 1). The particles left the surface at discrete times. The observed particles ranged in size from $<1$ to $\sim 10 \mathrm{~cm}$. The ejection locations occurred over a range of latitudes from $75^{\circ} \mathrm{S}$ to $20^{\circ} \mathrm{N}$. Particle velocities ranged from 0.07 to at least $3.3 \mathrm{~m} \mathrm{~s}^{-1}$. The minimum kinetic energy of the ejected particles ranged from 8 to $270 \mathrm{~mJ}$, assuming that the particles have albedos equivalent to the surface average of Bennu. Smaller events occurred that ejected fewer than 20 observed particles. Individual particles were ejected at a range of local solar times, including at night.

Dust ejection is a common phenomenon in comets and active asteroids. Even for wellstudied comets such as $67 \mathrm{P} /$ ChuryumovGerasimenko, substantial uncertainty exists as to the physical mechanism through which particles are released from the surface (21). We consider multiple hypotheses for the particle ejection mechanism, evaluating their respective strengths and weaknesses. These include rotational disruption, electrostatic lofting, cometlike ice sublimation, phyllosilicate dehydration, thermally driven stress fracturing, meteoroid impacts, and secondary impacts.

\section{Rotational disruption}

Mass shedding or splitting that results from rotational instability has been identified as a 

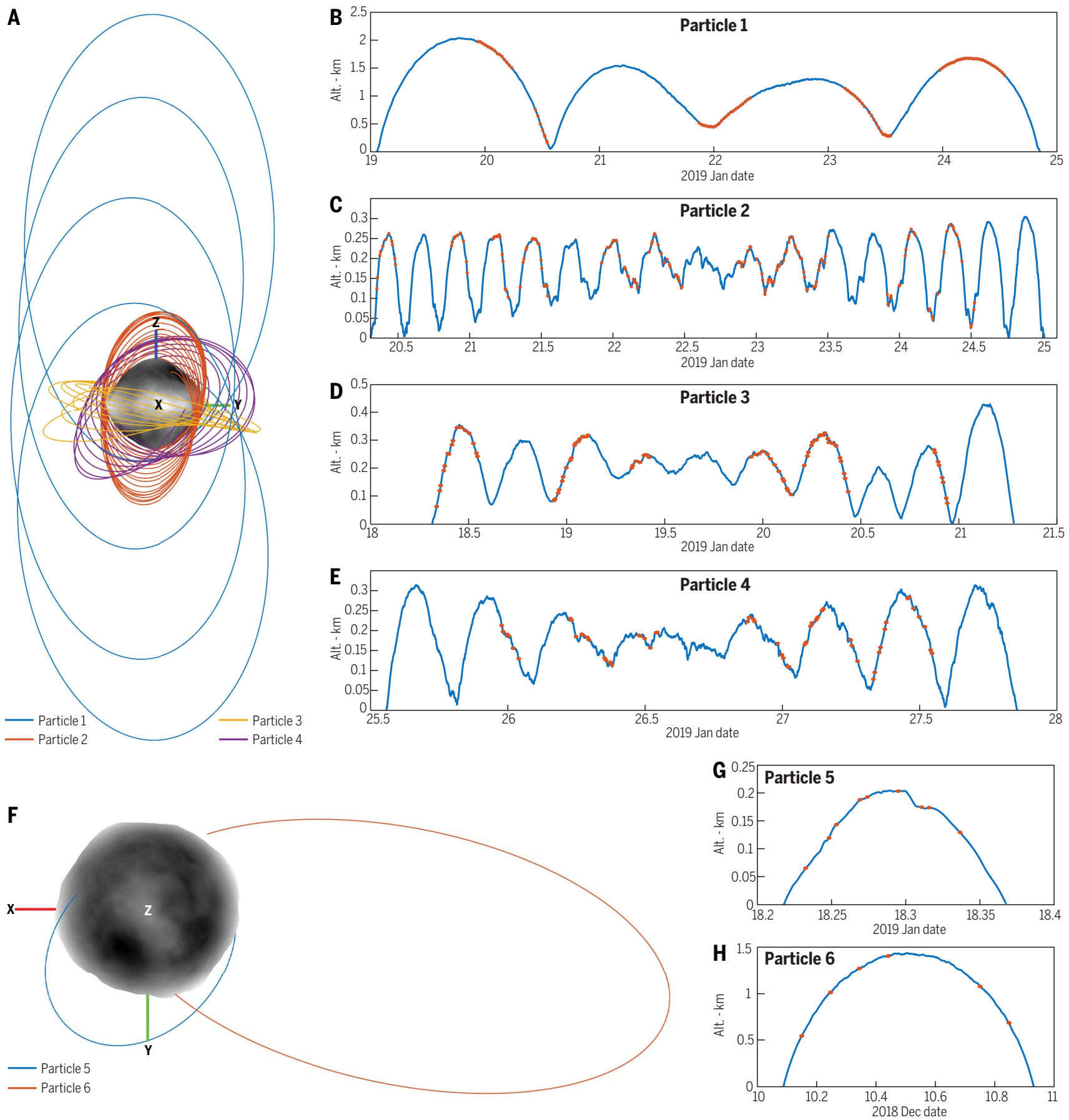

Fig. 4. Gravitationally bound particle altitudes and trajectories. (A) Trajectories and (B to $\mathbf{E})$ altitude above Bennu's surface over time for four orbiting particles (particles 1 to 4) (fig. S7 and tables S4 and S5). On the altitude plots, red circles mark the times of observations used in the trajectory estimate. Axis scales of the altitude plots differ. For particles 2 and 3 , it is not clear whether the last revolution depicted occurred or whether the particle impacted at the previous periapsis passage. The ragged appearance of the curves is a result of the rough topography of the surface of Bennu. $(\mathbf{F})$ Trajectories and ( $\mathbf{G}$ and $\mathbf{H}$ ) altitude above Bennu's surface over time, as in (A) to (E) but for two suborbital particles (particles 5 and 6) (fig. S7 and tables S4 and S5). The trajectories are seen from above Bennu's north pole $[x$ axis toward the Sun, $z$ axis close to Bennu's north (positive) pole, $y$ axis roughly in the direction of Bennu's heliocentric velocity]. Particle 6 is the earliest evidence of a particle in imaging data (10 December). possible explanation for the activity of the smaller active asteroids (22). In this scenario, rapidly rotating asteroids experience centrifugal forces greater than the centripetal forces from self-gravity, leading to particle ejection preferentially from low latitudes. Particles launched from Bennu's surface would have a maximum velocity equal to the equatorial surface velocity of $10 \mathrm{~cm} \mathrm{~s}^{-1}$ (on the basis of Bennu's $\sim 250$-m equatorial radius and $\sim 4.3$-hour rotation period). This mechanism would preferentially produce particles in equatorial orbits in the rotational direction. It is not capable of launching particles on retrograde or hyperbolic trajectories, as we observed. 

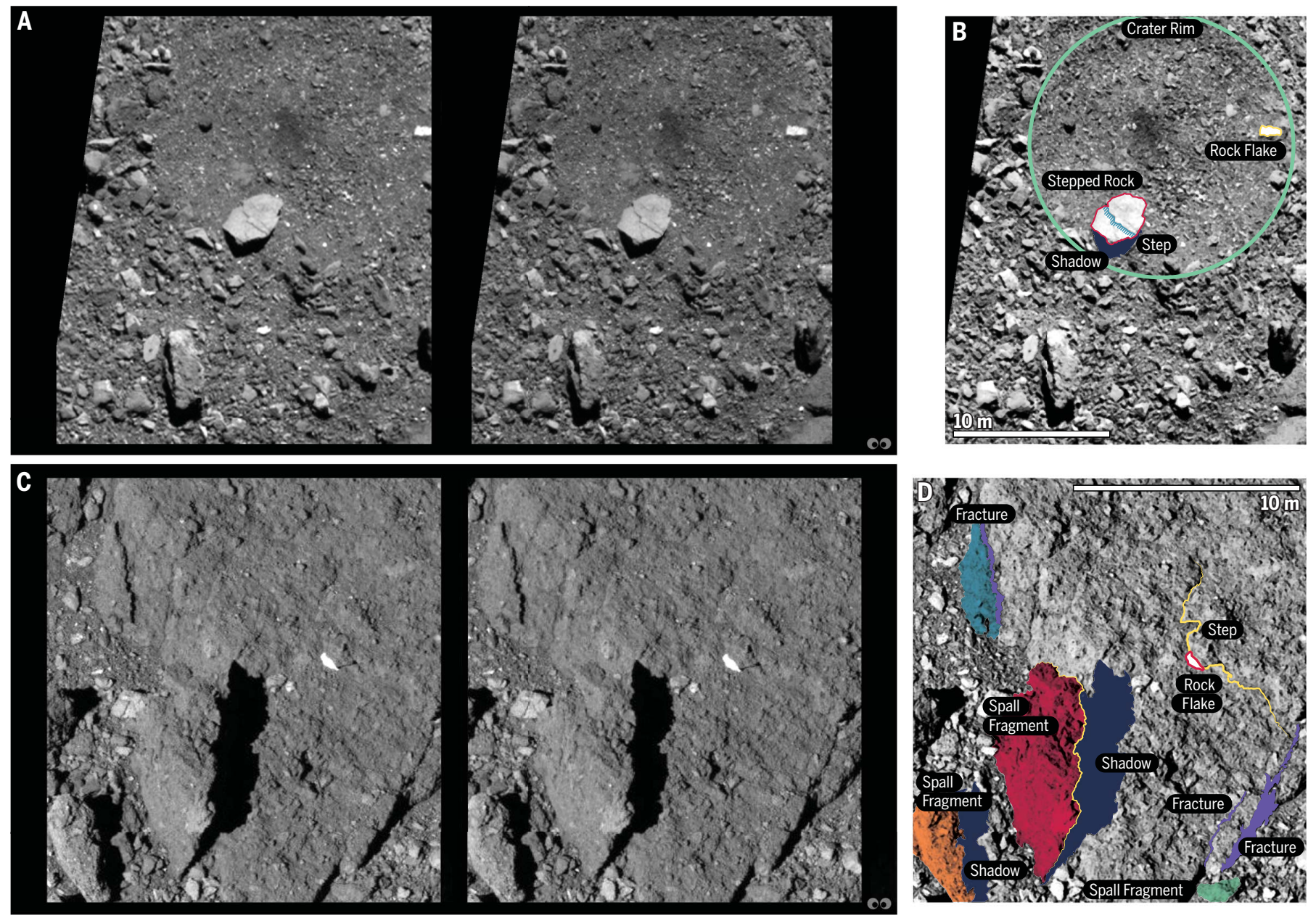

Fig. 5. Two distinct types of exfoliation textures on Bennu. In all images, north on Bennu is down. The PolyCam telescopic imager (12) acquired the four frames in $(A)$ and $(C)$ while the spacecraft moved with respect to the surface at a speed of $9 \mathrm{~cm} \mathrm{~s}^{-1}$ with exposures of (A) 1/300 of a second and (C) 1/200 of a second. These side-by-side stereo images are presented in the stereo "cross-eyed" configuration. A stereoscope-viewing version is available in fig. S10. Each pair of images has been adjusted to match their brightness, contrast, and shadow positions. (A) The parallax angle between these two images is $12^{\circ}$. Phase angle, $44^{\circ}$; pixel scale, $6.6 \mathrm{~cm}$ per pixel; (longitude, latitude), $\left(90^{\circ}, 11^{\circ}\right)$. (B) Annotated version of the image on the right in (A). The large, 5-m white rock on the crater rim displays a flat face, with a well- defined step crossing its center. A white "flake" is present in the upper right. (C) The parallax angle between these two images is $8^{\circ}$. Phase angle, $30^{\circ}$; pixel scale,

$4.7 \mathrm{~cm}$ per pixel; (longitude, latitude), $\left(44^{\circ},-30^{\circ}\right)$. (D) Annotated version of the image on the right in (C). The large black boulder displays exfoliation textures along both the east and west faces, with fractures running parallel to the texture in the rock.

The large rock column in the bottom left has a profile that matches that of the step in the boulder, suggesting that this fragment may have been uplifted in an energetic exfoliation event. Even though the rock slab measures 5 by 5 by $1 \mathrm{~m}$, it would only require $\sim 5 \mathrm{~J}$ of energy to lift it, assuming a density of $2 \mathrm{~g} \mathrm{~cm}^{-3}$. Other spalled fragments are present around the base of the large boulder.

\section{Electrostatic lofting}

Electrostatic lofting is the phenomenon of dust particles detaching from a surface once the electrostatic force on the particles exceeds those of gravity and cohesion (which bind the particles to the surface). The surface of an airless body (such as the Moon or an asteroid) interacts directly with the solar wind plasma, which charges the particles and produces a near-surface electric field. The electrostatic force is the product of the grain charge and the local electric field. Although electrostatic lofting has been discussed as a possible mechanism of the lunar horizon glow (23), when considering cohesion, there remained a discrepancy between the electrostatic force necessary to loft particles and the charging conditions hypothesized to be present in situ (24). Charge exchange between individual particles may produce very strong, short-scale electric fields that are capable of lofting particles in microgravity environments $(25,26)$. It is possible to electrostatically loft particles up to millimeters in radius at small asteroids such as Bennu (27), smaller than those we observed. The velocities of electrostatically lofted particles are likely to be less than $1 \mathrm{~m} \mathrm{~s}^{-1}$, unless additionally accelerated away from the surface by solar radiation pressure (27).

\section{Ice sublimation}

Dust release from comets is a major source of interplanetary dust particles. On comets, ice sublimation results in gas drag forces that eject dust particles from the surface (21). The gas-drag forces accelerate the released dust within a few times the radius of the nucleus, until solar radiation pressure takes over. For such sublimation to be the driver of the Bennu events, ice must be present at or near the surface. Several observed ejection events occurred at relatively low latitudes, where temperatures reach $\sim 390 \mathrm{~K}(17)$. At these temperatures, major cometary ice species $\left(\mathrm{CO}, \mathrm{CO}_{2}\right.$, and $\left.\mathrm{H}_{2} \mathrm{O}\right)$ are not stable [for example, (28)]. Additionally, there are no water-ice absorption features at 1.5 or $2.0 \mu \mathrm{m}$ in spectra of the surface (20). Subsurface ice could be trapped at depths greater than $1 \mathrm{~m}$ at some locations for long periods (29). Rapid volatile release from such a reservoir would require exposure by large 


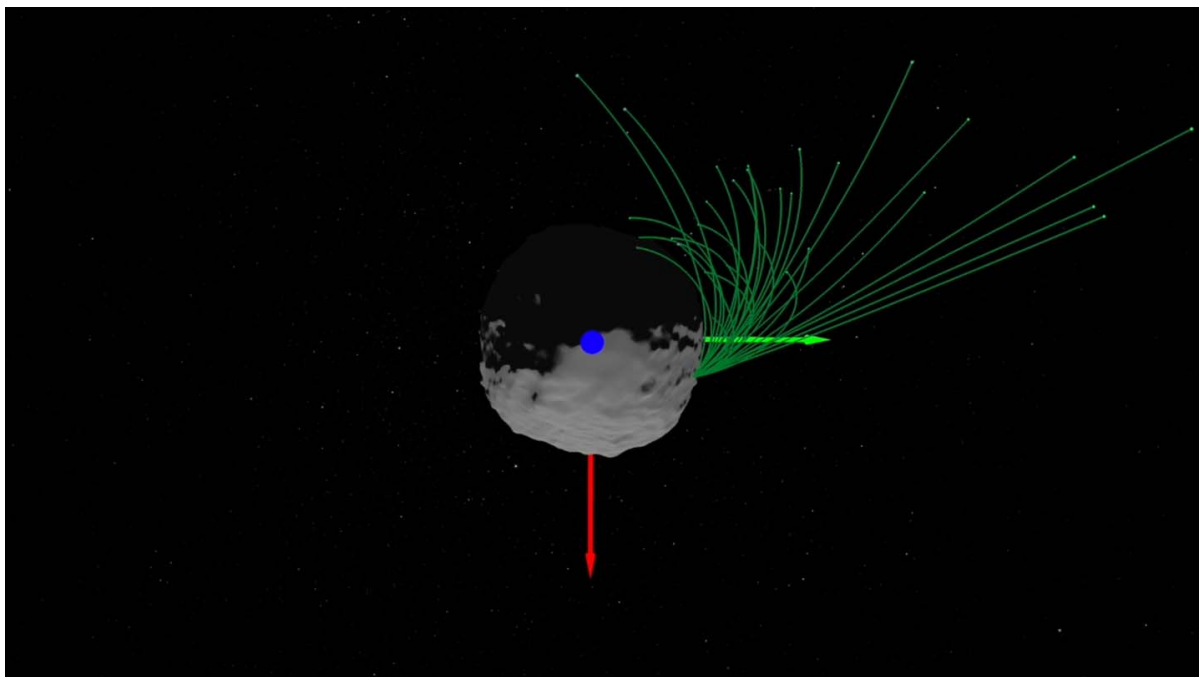

Movie 1. Animation showing the results of the orbit analysis for a subset of the particles ejected from Bennu on 19 January. The highest-velocity particles are on escape trajectories and leave the Bennu environment. Most of the particles are on suborbital trajectories and reimpact the surface, primarily on the night side of the asteroid.

impacts or deep thermal cracking at meter scales. We observed no geologic evidence of such processes acting recently at the event locations (Fig. 3). There is also no evidence of a coma or jets associated with volatile release (Fig. 1 and figs. S3 and S4).

\section{Phyllosilicate dehydration}

Although ice has not been observed on Bennu, the surface is rich in water-bearing minerals. Spectroscopy has shown that the surface is dominated by hydrated phyllosilicates, with the closest spectral match being CM-type carbonaceous chondrite meteorites (20). Evolved gas analysis experiments on Murchison (a CM chondrite) have demonstrated that considerable volatile release can occur when heated from ambient temperature up to $473 \mathrm{~K}$ under vacuum [for example, (30-32)]. Although this temperature is $\sim 70 \mathrm{~K}$ higher than the peak temperatures on Bennu, such low-temperature water release from Murchison indicates that the thermal dehydration of minerals begins with the loss of weakly bound adsorbed and interlayer water.

Mechanical stresses on Bennu's surface may generate adsorbed water, such as that released in laboratory experiments. The $\mathrm{CM}$ chondrites are dominated by Mg-rich serpentine and cronstedtite, an Fe-rich phyllosilicate [for example, (33)]. In these hydrated phases, particle size reduction through grinding enhances dehydroxylation and yields highly disordered material (34). The dehydroxylation reaction is substantially accelerated owing to the transformation of structural hydroxyls into adsorbed water in the resulting matrix. If mechanical stresses on Bennu result in a similar chemical transformation, the structural $\mathrm{OH}$ component of the phyllosilicates that dominate the surface mineralogy may be converted into absorbed water concentrated within an outer layer of the surface rocks. It is possible that the release of this adsorbed water within cracks and pores in boulders could provide a gas pressure leading to disruption of rock faces, such as is thought to occur on near-Earth asteroid (3200) Phaethon (35).

\section{Meteoroid impacts}

Solid bodies in space are routinely impacted by a steady flux of small meteoroids. Because Bennu is on an Earth-like orbit, we expect the flux of meteoroids at Bennu's surface to be similar to that on Earth, once corrected for gravitational focusing. A model of the interplanetary dust flux in near-Earth space has been determined by using data from in situ spacecraft measurements and lunar microcrater studies (36) and is widely adopted for meteoroid flux in near-Earth space (37). Lunar meteoroids typically impact at velocities between 13 and $18 \mathrm{~km} \mathrm{~s}^{-1}$ (38). If we assume an average velocity of $15.5 \mathrm{~km} \mathrm{~s}^{-1}$ for meteoroids at Bennu, an impact by an interplanetary dust particle with mass $2.5 \mu \mathrm{g}$ would deposit $300 \mathrm{~mJ}$ of energy into the surface, which is consistent with the estimated energy of the largest observed event (6 January). However, Bennu has a cross-sectional area of $1.96 \times$ $10^{5} \mathrm{~m}^{2}$; applying this value to the interplanetary dust flux model (36), we found that Bennu should be hit by a particle of this size on average once every minute, which is much more frequently than the observed ejection cadence. The large ejection events occurred on a roughly 2-week cadence. At that frequency, Bennu should be hit by an average of one meteoroid with a mass $\sim 3000 \mu \mathrm{g}$, depositing more than $360,000 \mathrm{~mJ}$ of energy into the surface if it impacted at $15.5 \mathrm{~km} \mathrm{~s}^{-1}$. Thus, only $0.07 \%$ of the impact energy from such events would need to be transferred to the particles to produce the largest observed ejection event.

The result of hypervelocity impacts into Bennu's surface depends substantially on the mass and velocity of the impacting grain and on the strength of the target material. Particle impacts at velocities on the order of 2.5 to $3 \mathrm{~km} \mathrm{~s}^{-1}$ produce well-developed craters with rims, fracturing, and spallation of a large number of particles (39). At higher speeds, such impact events produce little ejecta; instead, they deposit energy into a small volume of the asteroid surface, causing melting, vaporization, and at the highest energy densities, ionization of the target and impactor material producing plasma $(40,41)$. It is possible that the observed ejection events are the result of low-velocity meteoroid impacts, which occur much less frequently. Alternatively, the particles may be accelerated by the small fraction of impact energy from more frequent, highvelocity impacts that did not result in plasma production.

\section{Thermal stress fracturing}

Bennu's surface experiences extreme temperature variations over its 4.3-hour rotation period. Laboratory studies (42) showed that the CM chondrite Murchison quickly developed cracks and spalled particles from diurnal temperature cycling under near-Earth asteroid surface conditions. At the mid-latitudes, where the 19 January and 11 February events occurred, the surface temperature plunges to $250 \mathrm{~K}$ in the predawn hours and reaches a peak of $400 \mathrm{~K}$ at $\sim 13: 00 \mathrm{LST}$ (12). Because Bennu has a moderate thermal inertia of $350 \mathrm{~J} \mathrm{~m}^{-2} \mathrm{~K}^{-1} \mathrm{~s}^{-1 / 2}$ (17), the maximum temperature at the thermal skin depth (penetration depth of daily thermal conduction) of $\sim 2 \mathrm{~cm}$ occurs later in the afternoon, at 16:00 LST. The amplitude of temperature variation falls by a factor of $e$ at one thermal skin depth. Thus, for a region on Bennu whose maximum surface temperature is $400 \mathrm{~K}$, the peak temperature at a depth of $\sim 2 \mathrm{~cm}$ reaches $325 \mathrm{~K}$, inducing a strong thermal gradient over this short distance that cycles every 4.3 hours.

Thermal cycling can drive the growth of cracks in rocks over a range of spatial scales within the thermal skin depth, controlled by the amplitude and frequency of the temperature cycle, mineral composition, constituent grain size, the overall rock shape, and its orientation relative to the Sun. At the bulk scale, stresses associated with temperature gradients and surface cooling are induced in different regions of a boulder at different times throughout the thermal cycle. Stresses that arise in the 
shallow interior of large boulders tend to drive surface-parallel crack propagation (43). In the thermal fatigue regime, subcritical crack growth occurs slowly, propagating fractures incrementally over many cycles. Crack propagation velocity increases with crack length, until catastrophic disruption occurs, which may disaggregate material and eject particles from the surface.

In terrestrial settings, thermal fatigue combined with tectonic unloading is known to cause rock dome exfoliation and energetic particle ejection (44). In these studies, rocks show the greatest evidence for stress and microfracturing in the afternoon and evening. Although the tectonic unloading effects, which are not likely to be present on Bennu, are thought to add to the energy in these events, much less energy is needed to eject particles in a microgravity environment. Such energy may be stored as a result of structural deformation related to thermal strain, providing excess energy that leads to particle ejection (35).

\section{Secondary surface impacts}

A possible mechanism for the small ejection events is the reimpact of disaggregated material released by larger events. Analysis of particle trajectories in the largest events show that the particles have a substantial velocity component in the direction of asteroid rotation. Because the largest events occur in the afternoon, a large fraction of the particles on suborbital trajectories impact the night side of the asteroid (Movie 1). During impact, these particles may bounce off the surface or collide with other small particles on the surface, resulting in subsequent ejection of a small number of low-velocity particles.

Dynamical calculations show that ejecta moving at surface-relative velocities up to
$30 \mathrm{~cm} \mathrm{~s}^{-1}$ (escape velocity of $\sim 20 \mathrm{~cm} \mathrm{~s}^{-1}$ plus Bennu's surface rotational velocity of $10 \mathrm{~cm} \mathrm{~s}^{-1}$ ) lofted from the surface of Bennu can reimpact the surface days later (Movie 1). Depending on the impact location, reimpacting particles may be relaunched into a suborbital trajectory by bouncing off a hard surface such as a boulder (45) or ricocheting off a fine-grained surface $(46,47)$. Numerical simulations show that impacts on a fine-grained surface may result in the ejection of smaller surface particles at launch speeds that exceed the escape speed of Bennu (fig. S9). However, we have not directly observed particles ejecting from Bennu that are as large as the impactors in these simulations; in the energy regime that we have observed, particles of that size would not have traveled far enough from the asteroid to be detectable in our images. Our assessment thus leaves three viable candidates for the primary ejection mechanism: phyllosilicate dehydration, meteoroid impacts, and thermal stress fracturing (discussed in Conclusions and broader implications).

\section{Evidence from Bennu's geology}

Particle ejection from rock surfaces is consistent with the widespread observation of exfoliation features on Bennu's surface (Fig. 5 and fig. S10). Exfoliation is the division of a rock mass into lenses, plates, or parallel "sheets" because of differential stresses (48). For some bright boulders on Bennu (Fig. 5A), lineation is present on the rock faces, and they exhibit sheets that parallel the direction of fracture propagation. The more abundant dark boulders on Bennu also exhibit exfoliation (Fig. $5 \mathrm{C})$. In these rocks, the exfoliation fractures are linear, but the finer-grained texture appears as blocky segments in the fracture profile. Spalled fragments are seen resting on the surface and lying around the base of dark boulders.

The observed textures are characteristic of surface stresses that drive surface-perpendicular cracking, segment exfoliation sheets, and cause near-surface disaggregation. We do not observe similar spalled fragments in the immediate vicinity of the brighter boulders. However, we observe bright rocks perched on the surfaces of boulders, in orientations that exhibit no evident alignment with the underlying boulder's texture (for example, the bright object in the center-right of Fig. 5C). These bright rocks tend to have plate-like morphologies, similar to the exfoliation textures observed on the flat surfaces of the brighter rocks. Thus, exfoliation and fracturing may be operating on all boulders on Bennu, but the response of the bright rocks may be different-ejecting material over large distances, even on hyperbolic escaping trajectories, whereas the darker boulders decompose on site, creating a halo of spalled fragments.

\section{Implications for Bennu's geophysics}

The existence of low-energy particle ejection events on Bennu may result in reimpacting particles preferentially concentrated within the boundaries of Bennu's rotational Roche lobe (the region where material is energetically bound to the asteroid surface, between latitudes of $\sim \pm 23^{\circ}$ ) (16). A random distribution of ejection events with a sizable fraction of particle velocities less than the escape speed will preferentially transport material toward the equator owing to the lower geopotential. Once within the Roche lobe, the particles are trapped inside unless given a large enough speed (a few centimeters per second) and will be redistributed within the lobe owing to the chaotic orbital environment whenever lofted

Table 1. Characteristics of the three largest observed particle ejection events. The more extensive imaging datasets acquired for the 19 January and 11 February 2019 events, relative to that for the 6 January 2019 event, allowed higher-fidelity OD determination of the event locations and times. More detail is given in (12) and tables S2 and S3.

6 January

124 (of 200 total observed)
0.07 to 3.3
$<1$ to $8 \pm 3$
$270(+150 /-225)$

Minimum event energy $(\mathrm{mJ}+1 \sigma)$

Event location

Latitude (degrees, $\pm 3 \sigma$ )

Longitude (degrees, $\pm 3 \sigma$ )

Local solar time $( \pm 3 \sigma)$

UTC time $( \pm 3 \sigma)$

Near radiant
-74.95
$(+12.65 /-2.79)$
325.32
$(+18.91 /-10.28)$
$15: 22$
$+01: 06,-00: 36)$
$20: 58: 28$
$\pm 00: 00: 47$

Near radiant

12.65/-2.79)

8.91/-10.28)

01:06, -00:36)

\pm 00:00:47
19 January

93 (of 93 total observed)
0.06 to 1.3
$<1$ to $7 \pm 3$
$100(+50 /-85)$

OD radiant

20.63

$\pm 0.30$

335.40

$\pm 0.09$

16:38:01

\pm 00:00:23

00:53:41

\pm 00:00:04
11 February 60 (of 72 total observed) 0.07 to 0.21

$<1$ to $7 \pm 3$

$8(+4 /-7)$

OD radiant

20.68

$\pm 0.37$

60.17

$\pm 0.08$

18:05:31

\pm 00:00:22

23:27:28

\pm 00:00:06 
(16). Particles ejected with higher energies that achieve orbit will preferentially reimpact in the equatorial region within the lobe because of the larger asteroid radius there [(16), figure 5 therein]. After impact (which occurs at low speeds relative to the escape velocity), the particles will not have sufficient energy to escape the Roche lobe and again will be preferentially trapped, leading to a concentration of returning particles in these regions, as opposed to a globally uniform distribution.

Previous observations have indicated a steady increase in Bennu's rotation rate that will lead to doubling of that rate in $\sim 1.5$ million years; this acceleration is consistent with the Yarkovsky-O'Keefe-Radzievskii-Paddack (YORP) effect $(10,49)$. The angular momentum associated with particles ejected on escaping trajectories could also influence the rotation rate. It is possible to generate the measured rotational acceleration of Bennu by ejecting several particles of diameter $\sim 10 \mathrm{~cm}$ once per day in the westward direction from the equator, assuming no concurrent water vapor loss. A random ejection of escaping particles from the surface of a spinning body would produce a spin deceleration (50).

We summed the net angular momentum change from particles launched normal to every facet on the asteroid surface and given a sufficient ejection speed for escape $(12,16)$. We found that such a flux would always cause Bennu to spin slower (fig. S11), counteracting the YORP effect (50). This implies that the strength of the YORP effect on Bennu due to solar photons could be greater than originally estimated $(10,49)$. If Bennu were to eject, for example, on the order of $2010-\mathrm{cm}$ particles per day at a speed of $18 \mathrm{~cm} \mathrm{~s}^{-1}$ (the speed at which the effect is the greatest) normal to random points on its surface, then on average, its rotational acceleration would be slowed by less than $1 \%$ of the measured rotational acceleration. Thus, when averaged over the entire surface, the net effect of particle ejection is negligible relative to the YORP effect.

The linear momentum transfer from the particle ejections is orders of magnitude lower than that of the transverse acceleration because of thermal emission from Bennu, the operative component for the Yarkovsky effect (51). This acceleration peaks at $\sim 10^{-12} \mathrm{~m} \mathrm{~s}^{-2}$ during perihelion (51). Such an acceleration leads to a daily change in velocity $\Delta V$ of $10^{-7} \mathrm{~m} \mathrm{~s}^{-1}$, which is more than 7000 times the $\Delta V$ caused by a single 10 -cm particle with a density of $2 \mathrm{~g} \mathrm{~cm}^{-3}$ escaping at $1 \mathrm{~m} \mathrm{~s}^{-1}$.

\section{Conclusions and broader implications}

The ejection events on Bennu inform our understanding of active asteroids. There are substantial differences between active asteroids as commonly defined-where major mass loss events occur through processes such as large impacts, volatile release, and rotational acceleration, leading to mass shedding-and relatively small mass loss events as we see on Bennu. It is likely that there is a continuum of event magnitudes and that we have been limited to observing only the largest phenomena.

Mass loss observed during perihelion from the B-type near-Earth asteroid (3200) Phaethon, the parent body of the Geminids meteor shower, apparently consists of smaller particles $[1 \mu \mathrm{m}$ (52)] than observed at Bennu ( $<1$ to $\sim 10 \mathrm{~cm})$. However, particles in the centimeter size range were not observable during studies of Phaethon at perihelion, and sub-centimeter particles would have been difficult to detect in NavCam 1 images. Particles in the millimeter size range are observed as Geminids meteors (53). The mass loss from Bennu between 31 December and 18 February (including the three largest ejection events characterized above) was $\sim 10^{3} \mathrm{~g}$, which is orders of magnitude less than Phaethon's near-perihelion mass loss $\left(\sim 10^{4}\right.$ to $10^{5} \mathrm{~kg}$ per perihelion passage) (7). The mass loss rate $\left(\sim 10^{-4} \mathrm{~g} \mathrm{~s}^{-1}\right)$ on Bennu is also many orders of magnitude less than the rates observed at other active asteroids ( 10 to $\left.10^{3} \mathrm{~g} \mathrm{~s}^{-1}\right)$ (1). Mass loss as seen at Bennu suggests that Phaethon's current mass loss rate may include larger particles and be greater than remote observations imply.

Having evaluated multiple hypotheses for the mechanism of particle ejection on Bennu, we found that thermal fracturing, volatile release by dehydration of phyllosilicate rocks, and meteoroid impacts are plausible explanations. Rotational disruption and electrostatic lofting cannot explain the observed particle sizes and ejection velocities. There is no evidence for ice on the surface of Bennu or for recent exposure of a subsurface ice reservoir at the multiple ejection sites. Bennu's boulder morphology and the event ejection times are consistent with exfoliation as a result of thermal fracturing, phyllosilicate dehydration, or an interplay between these two mechanisms. Because we expect meteoroid flux to be greatest in the leading hemisphere (late afternoon on Bennu because of its retrograde rotation), the ejection event times are also consistent with meteoroid impacts. It is possible that multiple mechanisms operate in combination. Reimpacting particles could play a role in the smaller ejections or contribute to the larger events.

The particles that escape from Bennu on parabolic or hyperbolic orbits will escape onto heliocentric orbits, which we expect to disperse over time into a meteoroid stream. On the basis of the measured ejection velocities, meteoroids released after $1500 \mathrm{CE}$ would not have spread wide enough to bridge the current distance between the orbits of Bennu and Earth, 0.0029 AU, but will do so when that distance decreases later in the 21st century (54). However, if Bennu was active in the past, and the ejected particles survive for thousands of years, planetary perturbations would spread the stream wide enough to cause an annual meteor shower on present-day Earth around 23 September. The shower would radiate from a geocentric radiant at right ascension $5^{\circ}$, declination $-34^{\circ}$, and speed $6.0 \mathrm{~km} \mathrm{~s}^{-1}(54)$, corresponding to an apparent entry speed of $12.7 \mathrm{~km} \mathrm{~s}^{-1}$ (12). Meteoroids moving this slowly would create meteors of integrated visual magnitude +2 to -5 , assuming an $0.7 \%$ luminous efficiency (55). The stream would not easily blend with the sporadic background over thousands of years. No shower is detected in current meteor orbit survey data (56), but those data have poor coverage in the Southern Hemisphere.

The primary objective of OSIRIS-REx is to return samples of centimeter-scale rocks from the surface of Bennu to Earth for analysis (14). We have observed centimeter-scale particles frequently being ejected and reimpacting the asteroid surface. It is possible that the collected sample will contain some particles that were ejected and returned to Bennu's surface.

\section{REFERENCES AND NOTES}

1. D. Jewitt, H. Hsieh, J. Agarwal, "The active asteroids", in Asteroids IV, P. Michel, F. E. DeMeo, W. F. Bottke, Eds. (Univ. of Arizona Press, 2015), pp. 221-241.

2. E. W. Elst, G. Pizarro et al., Comet P/1996 N2 (ELST-PIZARRO). IAU Circular 6456 (1996).

3. D. Jewitt et al., Hubble Space Telescope investigation of main-belt comet 133P/Elst-Pizarro. Astron. J. 147, 117 (2014). doi: 10.1088/0004-6256/147/5/117

4. D. Jewitt et al., Episodically active asteroid 6478 Gault. Astrophys. J. 876, L19 (2019). doi: 10.3847/2041-8213/ab1be8

5. F. Moreno, J. Licandro, A. Cabrera-Lavers, F. J. Pozuelos, Early evolution of disrupted asteroid P/2016 G1

(PANSTARRS). Astrophys. J. 826, L22 (2016). doi: 10.3847/ 2041-8205/826/2/L22

6. J. Li, D. Jewitt, Recurrent perihelion activity in, (3200) Phaethon. Astron. J. 145, 154 (2013). doi: 10.1088/0004 $6256 / 145 / 6 / 154$

7. M.-T. Hui, J. Li, Resurrection of (3200) Phaethon in 2016. Astron. J. 153, 23 (2017)

8. D. Jewitt, The active asteroids. Astron. J. 143, 66 (2012). doi: 10.1088/0004-6256/143/3/66

9. D. S. Lauretta et al., The OSIRIS-REx target asteroid (101955) Bennu: Constraints on its physical, geological, and dynamical nature from astronomical observations. Meteorit. Planet. Sci. 50, 834-849 (2015). doi: 10.1111/maps.12353

10. C. W. Hergenrother et al., The operational environment and rotational acceleration of asteroid (101955) Bennu from OSIRIS-REx observations. Nat. Commun. 10, 1291 (2019). doi: 10.1038/s41467-019-09213-x; pmid: 30890725

11. M. G. Daly et al., The OSIRIS-REx Laser Altimeter (OLA) investigation and instrument. Space Sci. Rev. 212, 899-924 (2017). doi: 10.1007/s11214-017-0375-3

12. Materials and methods are available as supplementary materials.

13. B. J. Bos et al., Touch And Go Camera System (TAGCAMS) for the OSIRIS-REx asteroid sample return mission. Space Sci. Rev. 214, 37 (2018). doi: 10.1007/s11214-017-0465-2

14. D. S. Lauretta et al., OSIRIS-REx: Sample return from asteroid (101955) Bennu. Space Sci. Rev. 212, 925-984 (2017). doi: 10.1007/s11214-017-0405-1

15. B. Williams et al., OSIRIS-REx flight dynamics and navigation design. Space Sci. Rev. 214, 69 (2018). doi: 10.1007/s11214018-0501-x

16. D. J. Scheeres et al., The dynamic geophysical environment of (101955) Bennu based on OSIRIS-REx measurements. Nat. Astron. 3, 352-361 (2019). doi: 10.1038/s41550-019 0721-3 
17. D. N. DellaGiustina et al., Properties of rubble-pile asteroid (101955) Bennu from OSIRIS-REx imaging and thermal analysis Nat. Astron. 3, 341-351 (2019). doi: 10.1038/s41550-019-0731-1

18. K. J. Walsh et al., Craters, boulders and regolith of (101955) Bennu indicative of an old and dynamic surface. Nat. Geosci. 12, 399 (2019). doi: 10.1038/s41561-019-0360-4

19. D. S. Lauretta et al., The unexpected surface of asteroid (101955) Bennu. Nature 568, 55-60 (2019). doi: 10.1038/ s41586-019-1033-6; pmid: 30890786

20. V. E. Hamilton et al., Evidence for widespread hydrated minerals on asteroid (101955) Bennu. Nat. Astron. 3, 332-340 (2019). doi: 10.1038/s41550-019-0722-2; pmid: 31360777

21. J.-B. Vincent et al., Local manifestations of cometary activity. Space Sci. Rev. 215, 30 (2019). doi: 10.1007/s11214 019-0596-8

22. M. Drahus et al. Fast rotation and trailing fragments of the active asteroid P/2012 F5 (Gibbs). Astrophys. J. 802, L8 (2015). doi: 10.1088/2041-8205/802/1/L8

23. J. J. Rennilson, D. R. Criswell, Surveyor observations of lunar horizon-glow. Moon 10, 121-142 (1974). doi: 10.1007/BF00655715

24. C. M. Hartzell, D. J. Scheeres, The role of cohesive forces in particle launching on the moon and asteroids. Planet. Space Sci. 59, 1758-1768 (2014). doi: 10.1016/j.pss.2011.04.017

25. X. Wang, J. Schwan, H.-W. Hsu, E. Grün, M. Horányi, Dust charging and transport on airless planetary bodies. Geophys. Res. Lett. 43, 6103-6110 (2016). doi: 10.1002/2016GL06949

26. M. I. Zimmerman et al., Grain-scale supercharging and breakdown on airless regolith. J. Geophys. Res. Planets 121, 2150-2165 (2016). doi: 10.1002/2016JE005049

27. C. M. Hartzell, Dynamics of $2 \mathrm{D}$ electrostatic dust levitation at asteroids. Icarus 333, 234-242 (2019). doi: 10.1016/ j.icarus.2019.05.013

28. E. L. Andreas, New estimates for the sublimation rate of ice on the Moon. Icarus 186, 24-30 (2007). doi: 10.1016/ j.icarus.2006.08.024

29. N. Schorghofer, Predictions of depth-to-ice on asteroids based on an asynchronous model of temperature, impact stirring, and ice loss. Icarus 276, 88-95 (2016). doi: 10.1016/j.icarus.2016.04.037

30. E. K. Gibson Jr., Inorganic gas release studies and thermal analysis investigations on carbonaceous chondrites. Meteoritics 9. 343-344 (1974).

31. I. L. ten Kate et al., VAPoR-Volatile Analysis by Pyrolysis of Regolith-an instrument for in situ detection of water, noble gases and organics on the Moon. Planet. Space Sci. 58, 1007-1017 (2010). doi: 10.1016/j.pss.2010.03.006

32. E. K. Gibson Jr., S. M. Johnson, Thermogravimetric-quadrupole mass-spectrometric analysis of geochemical samples. Thermochim. Acta 4, 49-56 (1972). doi: 10.1016/0040-6031 (72)87062-X

33. D. S. Lauretta, X. Hua, P. R. Buseck, Mineralogy of fine-grained rims in the ALH 81002 CM chondrite. Geochim. Cosmochim. Acta 64, 3263-3273 (2000). doi: 10.1016/S0016-7037(00)00425-7

34. A. Drief, F. Nieto, The effect of dry grinding on antigorite from Mulhacen, Spain. Clays Clay Miner. 47, 417-424 (1999). doi: 10.1346/CCMN.1999.0470404

35. D. Jewitt, J. Li, Activity in geminid parent (3200) Phaethon Astron. J. 140, 1519-1527 (2010). doi: 10.1088/0004-6256/ $140 / 5 / 1519$

36. E. Grün, H. A. Zook, H. Fechtig, R. H. Giese, Collisional balance of the meteoritic complex. Icarus 62, 244-272 (1985). doi: 10.1016/0019-1035(85)90121-6

37. Committee for the Assessment of NASA's Orbital Debris Programs, Aeronautics and Space Engineering Board, Division on Engineering and Physical Sciences, Limiting Future Collision Risk to Spacecraft: An Assessment of Nasa's Meteoroid and Orbital Debris Programs (National Research Council, 2011).

38. H. A. Zook, "The state of meteoritic material on the Moon," in Lunar Science VI, 1301 (1975)

39. K. Fiege et al., Space weathering induced via microparticle impacts: 2. Dust impact simulation and meteorite target analysis. J. Geophys. Res. Planets 124, 1084-1099 (2019). doi: 10.1029/2018JE005564

40. M. S. Thompson, R. Christoffersen, T. J. Zega, L. P. Keller, Microchemical and structural evidence for space weathering in soils from asteroid Itokawa. Earth Planets Space 66, 89 (2014). doi: 10.1186/1880-5981-66-89
41. S. Drapatz, K. W. Michel, Theory of shock-wave ionization upon high-velocity impact of micrometeorites. Z. Naturforsch. A 29 870-879 (1974). doi: 10.1515/zna-1974-0606

42. M. Delbo et al., Thermal fatigue as the origin of regolith on small asteroids. Nature 508, 233-236 (2014). doi: 10.1038/ nature13153; pmid: 24695219

43. J. L. Molaro, S. Byrne, J.-L. Le, Thermally induced stresses in boulders on airless body surfaces, and implications for rock breakdown. Icarus 294, 247-261 (2017). doi: 10.1016/ j.icarus.2017.03.008

44. B. D. Collins et al., Thermal influences on spontaneous rock dome exfoliation. Nat. Commun. 9, 762 (2018). doi: 10.1038/ s41467-017-02728-1; pmid: 29472534

45. H. Yano et al., Touchdown of the Hayabusa spacecraft at the Muses Sea on Itokawa. Science 312, 1350-1353 (2006) doi: 10.1126/science.1126164; pmid: 16741113

46. C. Maurel, P. Michel, J. Biele, R.-L. Ballouz, F. Thuillet, Numerical simulations of the contact between the lander MASCOT and a regolith-covered surface. Adv. Space Res. 62 2099-2124 (2018). doi: 10.1016/j.asr.2017.05.029

47. F. Thuillet et al., Numerical modeling of lander interaction with a low-gravity asteroid regolith surface: Application to MASCOT onboard Hayabusa2. Astron. Astrophys. 615, A41 (2018). doi: 10.1051/0004-6361/201832779

48. G. R. Holzhausen, Origin of sheet structure, 1. Morphology and boundary conditions. Eng. Geol. 27, 225-278 (1989). doi: 10.1016/0013-7952(89)90035-5

49. M. C. Nolan et al., Detection of rotational acceleration of Bennu using HST lightcurve observations. Geophys. Res. Lett. 46, 1956-1962 (2019). doi: 10.1029/2018GL080658

50. A. R. Dobrovolskis, J. A. Burns, Angular momentum drain: A mechanism for despinning asteroids. Icarus 57, 464-476 (1984). doi: 10.1016/0019-1035(84)90130-1

51. S. R. Chesley et al., Orbit and bulk density of the OSIRIS-REX target Asteroid (101955) Bennu. Icarus 235, 5-22 (2014). doi: 10.1016/j.icarus.2014.02.020

52. D. Jewitt, J. Li, J. Agarwal, The dust tail of asteroid (3200) Phaethon. Astrophys. J. 771, L36 (2013). doi: 10.1088/20418205/771/2/L36

53. V. Vojáček, J. Borovička, P. Koten, P. Spurný, R. Štork, Properties of small meteoroids studied by meteor video observations. Astron. Astrophys. 621, A68 (2019). doi: 10.1051/ 0004-6361/201833289

54. Q. Ye, Prediction of meteor activities from (101955) Bennu. Res. Notes AAS 3, 56 (2019). doi: 10.3847/2515-5172/ab12e7

55. R. J. Weryk, P. G. Brown, Simultaneous radar and video meteors-II: Photometry and ionisation. Planet. Space Sci. 81, 32-47 (2013). doi: 10.1016/j.pss.2013.03.012

56. P. Jenniskens et al., CAMS: Cameras for Allsky Meteor Surveillance to establish minor meteor showers. Icarus 216 , 40-61 (2011). doi: 10.1016/j.icarus.2011.08.012

\section{ACKNOWLEDGMENTS}

We are grateful to the entire OSIRIS-REx Team for making the encounter with Bennu possible. Funding: This material is based upon work supported by NASA under Contracts NNM10AA11C and NNG13FC02C issued through the New Frontiers Program. A portion of this work was conducted at the Jet Propulsion Laboratory, California Institute of Technology, under a contract with NASA. OLA and funding for the Canadian authors was provided by the Canadian Space Agency. C.M.H., J.L.M., and P.T. acknowledge support from NASA's OSIRIS-REx Participating Scientist Program (grants 80NSSC18K0227. 80NSSC18K0239, and 80NSSC18K0280, respectively). P.M., G.L., and F.T. acknowledge funding support from the French Agency CNES and from the Academies of Excellence on Complex Systems and Space, Environment, Risk and Resilience of the Initiative d'EXcellence "Joint, Excellent, and Dynamic Initiative" (IDEX JEDI) of the Université Côte d'Azur. J.L. and J.d.L. acknowledge funding support from the projects AYA2015-67772-R (MINECO, Spain) and ProlD20170112 (ACIISI/ Gobierno de Canarias/EU/FEDER). B.Ro. acknowledges funding support from the Royal Astronomical Society (RAS) and the UK Science and Technology Facilities Council (STFC). Author contributions: D.S.L. led the scientific investigation and developed the hypotheses for ejection mechanisms. C.W.H. detected the particle ejection events and led the photometric modeling. C.K.M.
J.N.K.Jr., and J.Y.L. supported the photometric modeling efforts. S.R.C. led the team that performed the orbital element analysis of the six short-lived orbiting particles, supported by R.A.J., M.B., A.B.D. D.F. Y.T., W.M.O.Jr., D.J.S., and J.W.M. D.J.S. also calculated the Bennu Roche lobe and contribution of particle ejections to Bennu's observed rotational acceleration and Yarkovsky. M.C.M. led the team that performed the OpNav and OD analyses. J.M.L. performed the OD analysis for the three largest observed ejection events. J.Y.P. led the OpNav characterization of the three largest events, supported by A.J.L., E.J.L-C., C.D.A. D.S.N., L.K.M., and E.M.S. M.G.D. is the lead instrument scientist for OLA and performed the analysis of the off-body lidar returns, supported by M.A.A. and J.A.S. B.J.B. is the lead instrument scientist for NavCam 1 and performed image calibration and image processing for the navigation images. B.Ri. and C.Y.d.'A. are the lead and deputy lead instrument scientists, respectively, for the OSIRIS-REx Camera Suite; they analyzed images of Bennu's surface for evidence of particle infall and processed the images used for the stereo pairs. D.N.D. is the lead image processing scientist for OSIRIS-REx and prepared the global mosaics used to register the particle source locations, supported by K.J.B., C.A.B., and D.R.G. K.J.B. also developed the NavCam camera model for use in registering NavCam images relative to the Bennu shape model and the background star fields. J.P.E. is the lead thermal analysis scientist for OSIRIS-REx, and B.Ro. developed the asteroid thermal model to determine the surface temperatures, skin depths, and thermal gradients at the particle ejection sites and globally across the asteroid. R.-L.B. developed the secondary impact hypothesis with support from P.M. and F.T. W.F.B. provided input on the meteoroid impact hypothesis and evaluated the other hypotheses in the context of the dynamical evolution of Bennu. H.C., J.d.L., and J.L. provided expertise on other known active asteroids. H.C.C.Jr. provided input on the potential mechanisms for ejection events and on the content of the manuscript. J.P.D. provided rock count data for testing the reimpacting particle hypothesis. D.P.G. counted rocks and provided input on EGA studies of meteorites and their low-temperature volatile release. C.M.H. developed the electrostatic lofting hypothesis. P.J. developed the hypothesis on the potential Bennu meteor shower. E.R.J. performed the geologic analysis of the particle ejection source regions. G.L. provided input on the relevance of thermal cycling experiments and regolith evolution. C.M. and B.M. identified and processed the stereo pair images. J.L.M. provided input on the feasibility of thermal fracture processes as a mechanism for particle ejection. H.L.R. led the development of the figures. S.H.S. provided software for processing NavCam images and identifying candidate particles. P.T. performed calculations for the statistical assessment of particles lifetime and fallback distribution. D.V. supported the development of the force model for nongravitational forces for orbiting particles, as well as giving input on the general implications and context of Bennu's activity. C.W.V.W. contributed substantively to the writing and preparation of the manuscript. Competing interests: H.C.C.Jr. is also affiliated with the Department of Earth and Planetary Science, American Museum of Natural History, New York, NY, USA. J.L.M. is also affiliated as a contractor with the Jet Propulsion Laboratory, California Institute of Technology, Pasadena, CA, USA. Data and materials availability: NavCam 1 images from Orbital A and OLA data from Preliminary Survey are available from the Planetary Data System (PDS) at https://sbn.psi.edu/pds/resource/orex/tagcams. html and https://sbn.psi.edu/pds/resource/orex/ola.html, respectively. The NavCam 1 images that we used are listed in data file S1. The parameters of the three largest ejection events are given in tables S2 and S3, and the derived orbital data for the six gravitationally bound particles are in tables $\$ 4$ and $S 5$ and data file S1.

\section{SUPPLEMENTARY MATERIALS}

science.sciencemag.org/content/366/6470/eaay3544/suppl/DC1 Materials and Methods

Figs. S1 to S11

Tables S1 to S5

References (57-92)

Data File S1

11 June 2019; accepted 22 October 2019

10.1126/science.aay3544 


\section{Science}

\section{Episodes of particle ejection from the surface of the active asteroid (101955) Bennu}

D. S. Lauretta, C. W. Hergenrother, S. R. Chesley, J. M. Leonard, J. Y. Pelgrift, C. D. Adam, M. Al Asad, P. G. Antreasian, R.-L. Ballouz, K. J. Becker, C. A. Bennett, B. J. Bos, W. F. Bottke, M. Brozovic, H. Campins, H. C. Connolly Jr., M. G. Daly, A. B. Davis, J. de León, D. N. DellaGiustina, C. Y. Drouet d'Aubigny, J. P. Dworkin, J. P. Emery, D. Farnocchia, D. P. Glavin, D. R. Golish, C. M. Hartzell, R. A. Jacobson, E. R. Jawin, P. Jenniskens, J. N. Kidd Jr., E. J. Lessac-Chenen, J.-Y. Li, G. Libourel, J. Licandro, A. J. Liounis, C. K. Maleszewski, C. Manzoni, B. May, L. K. McCarthy, J. W. McMahon, P. Michel, J. L. Molaro, M. C. Moreau, D. S. Nelson, W. M. Owen Jr., B. Rizk, H. L. Roper, B. Rozitis, E. M. Sahr, D. J. Scheeres, J. A. Seabrook, S. H. Selznick, Y. Takahashi, F. Thuillet, P. Tricarico, D. Vokrouhlický and C. W. V. Wolner

Science 366 (6470), eaay3544

DOI: $10.1126 /$ science.aay3544

\section{Bennu ejects material from its surface}

Most asteroids appear inert, but remote observations show that a small number experience mass loss from their surfaces. Lauretta and Hergenrother et al. describe close-range observations of mass loss on the near-Earth asteroid Bennu (see the Perspective by Agarwal). Shortly after arriving at Bennu, navigation cameras on the OSIRIS-REx (Origins, Spectral Interpretation, Resource Identification, and Security--Regolith Explorer) spacecraft detected objects 1 to 10 centimeters in diameter moving above the surface. Analysis of the objects' trajectories showed that they originated in discrete ejection events from otherwise unremarkable locations on Bennu. Some objects remained in orbit for several days, whereas others escaped into interplanetary space. The authors suggest multiple plausible mechanisms that could underlie this activity.

Science, this issue p. eaay3544; see also p. 1192

ARTICLE TOOLS

SUPPLEMENTARY

MATERIALS

RELATED

REFERENCES

PERMISSIONS http://science.sciencemag.org/content/366/6470/eaay3544

http://science.sciencemag.org/content/suppl/2019/12/04/366.6470.eaay3544.DC1

http://science.sciencemag.org/content/sci/366/6470/1192.full file:/content

This article cites 80 articles, 2 of which you can access for free http://science.sciencemag.org/content/366/6470/eaay3544\#BIBL

http://www.sciencemag.org/help/reprints-and-permissions

Use of this article is subject to the Terms of Service

Science (print ISSN 0036-8075; online ISSN 1095-9203) is published by the American Association for the Advancement of Science, 1200 New York Avenue NW, Washington, DC 20005. The title Science is a registered trademark of AAAS.

Copyright (C) 2019 The Authors, some rights reserved; exclusive licensee American Association for the Advancement of Science. No claim to original U.S. Government Works 